\title{
Low frequency oscillations of the magnetosphere
}

\author{
S. Børve ${ }^{1,2}$, H. Sato ${ }^{3}$, H. L. Pécseli*4, and J. K. Trulsen ${ }^{1}$ \\ ${ }^{1}$ Institute of Theoretical Astrophysics, University of Oslo, Boks 1029 Blindern, N-0315 Oslo, Norway \\ ${ }^{2}$ Norwegian Defence Research Establishment (FFI), N-2027 Kjeller, Norway \\ ${ }^{3}$ German Aerospace Center (DLR), Institute of Communications and Navigation, D-17235 Neustrelitz, Germany \\ ${ }^{4}$ Department of Physics, University of Oslo, Boks 1048 Blindern, N-0316 Oslo, Norway ( hans.pecseli@fys.uio.no )
}

\begin{abstract}
Sudden pulses in the model solar wind sets the Earth's magnetosphere into damped oscillatory motions. A simple model is capable of explaining many of the basic properties of these oscillations, giving scaling laws for their characteristics in terms of the parameters of the problem, such as the Solar wind momentum density. The period of the oscillations, their damping and anharmonic nature are accounted for. The model has no free adjustable numerical parameters and can be seen as an effort to predict some dynamic properties of the magnetosphere on the basis of measurable steady state characteristics. A simple test of the model is found by comparing its prediction of the Earthmagnetopause distance with observed values. The results agree well with satellite observations and also numerical simulations.
\end{abstract}

\section{Introduction}

Observations indicate that sudden pulses in the model solar wind can set the Earth's magnetosphere into damped oscillatory motions. Oscillation periods on the order of 5-10 min have been observed by instrumented spacecrafts [1,2]. The damping rate can vary, but often it is found that the oscillations damp within a few periods of oscillations. Our analysis is based on a simple model for the contact between the Earth's magnetosphere and the highly conducting solar wind. Two questions are addressed: the distance between the Earth and the magnetopause for steady state conditions, and the dynamic response of the magnetopause position following an impulse in the solar wind. Such an impulse can have the form of a density increase or a sudden decrease in solar wind momentum density. Due to the nonlinearity of the interaction, the two responses are not trivially related. For the steady state distance $R$ from the Earth to the magnetopause we find analytically

$$
R=\left(\frac{\mu_{0} \mathcal{M}^{2}}{8 \pi^{2} n M U^{2}}\right)^{1 / 6},
$$

with $n M U$ being the solar wind momentum density and $n m U^{2}$ is the directed solar wind pressure, while $\mathcal{M}$ is the magnetic dipole moment of the Earth. Realistic parameters give distances in the range of 10 - 15 Earth radii, which agree well with observations. The model gives a characteristic period of oscillations

$$
T \equiv \frac{2 \pi}{\Omega}=\frac{2 \pi R}{U} \sqrt{\frac{D \rho}{6 n M R}},
$$

where $D$ is the thickness of the magnetosheath, and $\rho$ is the average mass density of the magnetosheath plasma, while $n M$ is the solar wind mass density. We find $T$ typically in the range of $10-30 \mathrm{~min}$, depending on parameters.

\section{Numerical results}

The analysis is supported by numerical simulations solving the Magneto Hydro Dynamic (MHD) equations in two spatial dimensions, where we let a solar wind interact with a magnetic dipole representing a magnetized Earth [3]. The numerical solutions are based on a Smooth Particle Hydrodynamics (SPH) code, assuming ideal MHD. Two tiltangles of the Earth's magnetic dipole axis were considered. In all cases analyzed, we observe the formation of a magneto sheath, with the magnetopause at a distance corresponding well to the analytical results. The analytical model seems to be robust, and gives good qualitative agreement with the numerical simulations for a range of parameters, also 
concerning oscillation periods and damping times for cases where the dynamic response to perturbations are considered. Illustrative results for the steady state magnetosphere are shown in Fig. 1, and the dynamic responses to pulsations in the solar wind are shown in Fig. 2.
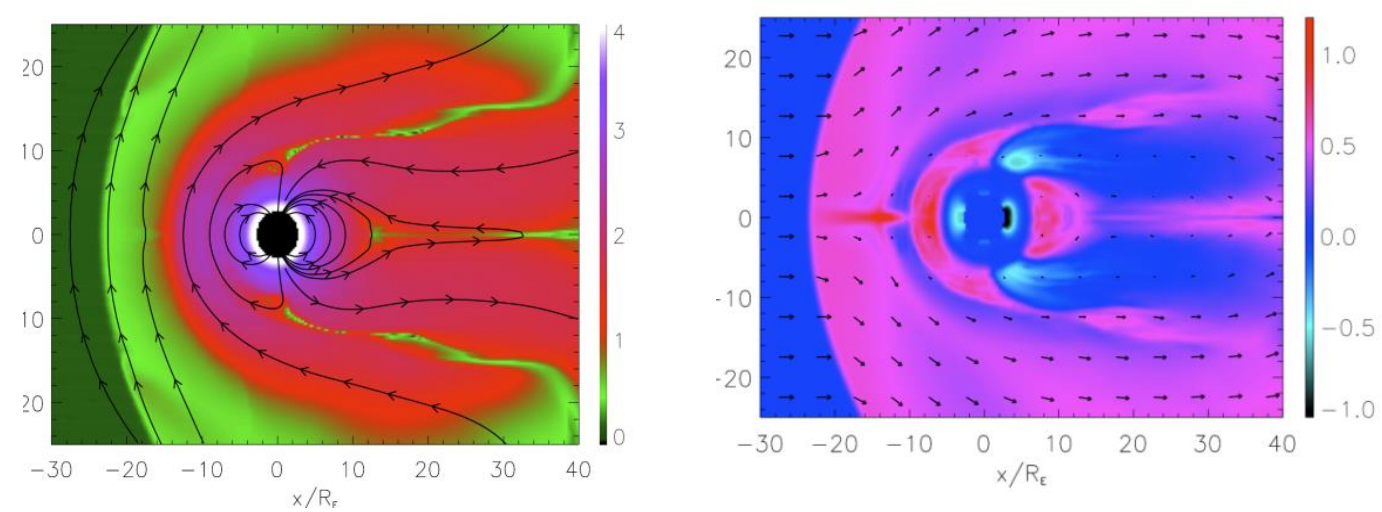

Fig. 1 The magnetic field intensity is shown to the left with color scale in arbitrary units, with small arrows giving the local field direction. The plasma density is shown to the right with small arrows showing the direction of the local plasma flow velocity. Distances are normalized by Earth radii, $R_{E}$.
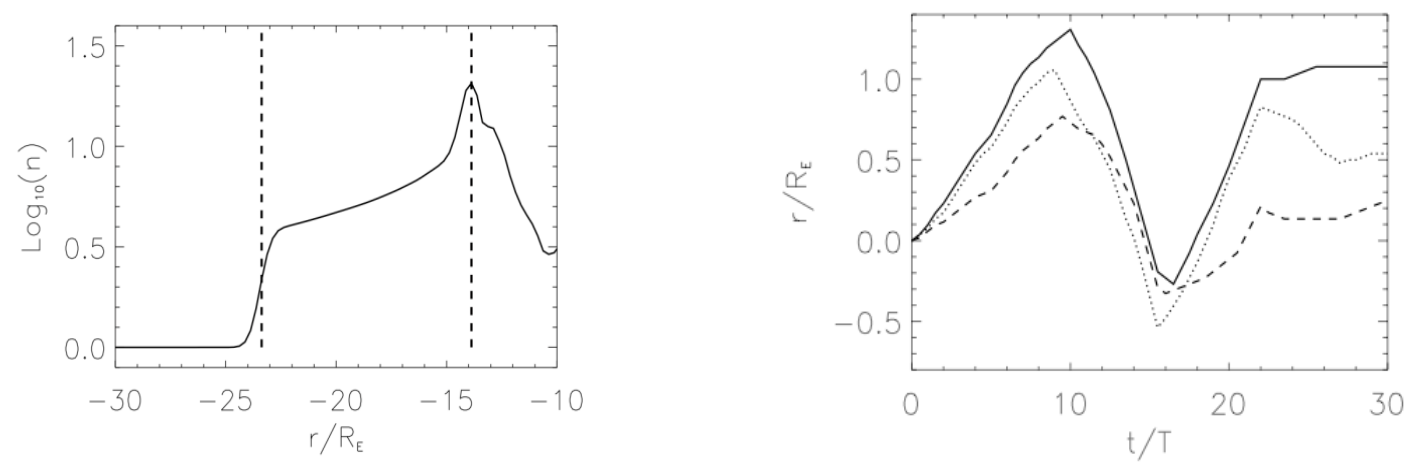

Fig. 2 Numerical results for the numerically obtained magnetosheath, see also Fig. 1. The vertical dashed lines indicate positions where the plasma motion is sampled. Note that the vertical axis is logarithmic, so that $\log (\mathrm{n})=0$ corresponds to the normalizing solar wind density. For this case we find $D \approx 9 R_{E}$. The figure to the right shows the oscillatory response to an impulse in the solar wind for relative density variations of $\Delta n / n=10 \%, 15 \%$ and $20 \%$.

The analysis allows also for predicting the magnetic field perturbations detected on Earth due to changes in the magneto sheath current. Basically, the perturbations can be explained by a superposition of suitably placed magnetic dipoles, and dynamic features accounted for by the relative motion of these dipoles. The results apply best for the part of the Earth's surface facing the Sun. The model is restricted to a space-time variation implied by the validity of magneto hydrodynamics (MHD). The model can be improved by taking effects due to magneto sheath curvature into account.

\section{References:}

1. C. J. Farrugia and F. T. Gratton, "Aspects of magnetopause/magnetosphere response to interplanetary discontinuities, and features of magnetopause Kelvin-Helmholtz waves”, J. Atmos. Solar-Terr.Phys., 73, 2011, pp. 40-51.

2. F. Plaschke, K.-H. Glassmeier, D. G. Sibeck, H. U. Auster, O. D. Constantinescu, V. Angelopoulos, and W. Magnes, "Magnetopause surface oscillation frequencies at different solar wind conditions", Ann. Geophys., 27, 2009, pp. 45214532 .

3. S. Børve, H. Sato, H. L. Pécseli, and J. K.. Trulsen, "Minute-scale period oscillations of the magnetosphere", Ann. Geophys., 29, 2011, pp. 663-671. 